\title{
The First Comprehensive Photometric Study of the Algol-type System CL Aurigae
}

\author{
Jae Woo Lee ${ }^{1}$, Chun-Hwey Kim² ${ }^{2}$, Duck Hyun Kim² ${ }^{2}$, Seung-Lee Kim ${ }^{1}$, Chung-Uk Lee ${ }^{1}$, and \\ Robert H. Koch ${ }^{3}$ \\ ${ }^{1}$ Korea Astronomy and Space Science Institute, Daejeon 305-348, Korea \\ jwlee@kasi.re.kr, slkim@kasi.re.kr, leecu@kasi.re.kr \\ ${ }^{2}$ Department of Astronomy and Space Science, and Chungbuk National University Observatory, \\ Chungbuk National University, Cheongju 361-763, Korea \\ kimch@chungbuk.ac.kr, kdh3841@hanmail.net \\ ${ }^{3}$ Department of Physics and Astronomy, University of Pennsylvania, Philadelphia, USA \\ rhkoch@earthlink.net
}

\begin{abstract}
We present the first extensive photometric results of CL Aur from our BVRI CCD photometry made on 22 nights from 2003 November through 2005 February. Fifteen new timings of minimum light were obtained. During the past 104 years, the orbital period has varied due to a periodic oscillation superposed on a continuous period increase. The period and semi-amplitude of the oscillation are about $21.6 \mathrm{yrs}$ and $0.0133 \mathrm{~d}$, respectively. This detail is interpreted as a light-travel-time effect due to a low-luminosity K-type star gravitationally bound to the CL Aur close system. Our photometric study indicates that CL Aur is a relatively short-period Algol-type binary with values of $q=0.602$ and $i=88^{\circ} .2$. Mass transfer from the secondary to the primary eclipsing component is at least partly responsible for the observed secular period change with a rate of $d P / d t=+1.4 \times 10^{-7} \mathrm{~d} \mathrm{yr}^{-1}$. A cool spot model has been calculated but we think that an alternative hot-spot model resulting from a gas stream impact on the hot star is more reasonable despite two difficulties with the explanation. Absolute dimensions of the eclipsing system are deduced and its present state is compared with tracks for single star and conservative close binary evolution. Finally, we examine the possible reconciliation of two different calculations of the luminosity of the hot spot and a reinterpretation of the secular term of the period variability.
\end{abstract}

Subject headings: binaries: close — binaries: eclipsing — stars: individual (CL Aurigae) — stars: spots 


\section{INTRODUCTION}

Algol-type close binaries are semi-detached interacting systems in which one type of interaction is mass transfer between the component stars by means of a gas stream. They have been known as good astrophysical laboratories for studying accretion processes because a number of them are bright. They are in the slow phase of mass transfer with $d M / d t \simeq 10^{-11}-10^{-7} \mathrm{M}_{\odot} \mathrm{yr}^{-1}$ and do not undergo violent eruptions that interfere with the accretion process. The circumstellar structures produced by the mass-transfer process in these systems have been sorted according to orbital period by Richards \& Albright (1999) but do not depend upon it significantly. Rather, their natures can be easily understood from the position of the mass-gaining component in the so-called $r-q$ diagram in which the fractional radius $r=(R / a)$ of a gainer is plotted versus the mass ratio $q$ and compared with the semianalytical computations of the gas stream hydrodynamics of Lubow \& Shu (1975). In the short-period Algols located above the $\omega_{d}$ curve of the diagram (cf. Figure 2 of Richards \& Albright), the hot, detached primary star is large relative to the orbital radius and the two components are too close to each other to form an accretion disk or even a stable accretion annulus. Instead, it is possible that an impact region, and hence a hot spot, can be formed on the surface of the primary star somewhat displaced from the line of centers due to the Coriolis acceleration imposed on the flowing gas. If the secondary stars are sufficiently cool, they likely display enhanced magnetic activity due to deep outer convective layers and rapid rotation. This magnetic mechanism may contribute to the period and light variations for systems with spectra later than F-type (Hall 1989).

CL Aur (GSC 2393-1455, HV 6886, TYC 2393-1455-1) was discovered to be a variable star by Hoffleit (1935) based on photographic plate estimates. Kurochkin (1951) presented the first (partial) photographic light curve of the star and the original light elements, Min. I = HJD 2,432,967.262+ 1.2443666E. The value of the period positions this object toward the short-period limit for Algols. The spectral type of the primary star was classified to be A0 by Götz \& Wenzel (1968). Since then, times of minimum light have been published assiduously by numerous workers but, to our knowledge, a complete light curve and the fundamental parameters for the binary system have not been made so far. Changes of the orbital period have been considered by Hegedüs (1988) and Wolf et al. (1999). Hegedüs selected this system as a possible candidate for the study of apsidal motion. However, the later authors ruled out this possibility from CCD timings for primary and secondary eclipses. They suggested the cause of period variation to be a light-travel-time (LTT) effect due to the presence of a third body in the binary system. Most recently, Wolf et al. (2007, hereafter W07) reported that a long-term period increase is superimposed on an LTT orbit with a period of $P_{3}=21.7 \mathrm{yrs}$, a semi-amplitude of $K=0.014 \mathrm{~d}$, and an eccentricity of $e=0.32$.

In the Simbad data base 1 , the system is described as an eclipsing binary of $\beta$ Lyr type. $B V J H K$ magnitues are listed for the star but these are from heterogeneous sources and are not mu-

\footnotetext{
${ }^{1}$ http://simbad.u-strasbg.fr/simbad/
} 
tually consistent. Part of this inconsistency arises because the magnitudes refer to different phases in the Keplerian cycle. With the well-known transformations (ESA 1997), standard photometric values for CL Aur in the Johnson system were calculated to be $V=+11.62$ and $(B-V)=+0.33$ from the Tycho results. These refer to some unknown Keplerian phase. These are not consistent with those in Simbad presumably because of the large phase-locked variations in magnitude and color index of the binary.

At present, CL Aur is known only as a neglected eclipsing system composed of an A-type primary and a cooler companion. In order to derive photometric solutions and to examine whether the W07 suggestion is appropriate for the orbital period change, we decided to obtain light curves with multiband photometry. In this paper, we present the first mutual analyses of the $O$ - $C$ diagram and the light curves.

\section{CCD PHOTOMETRIC OBSERVATIONS}

New photometric observations of CL Aur were obtained using a SITe 2K CCD camera and a $B V R I$ filter set attached to the 61-cm reflector at Sobaeksan Optical Astronomy Observatory (SOAO) in Korea. The observations of the first season were made on 14 nights from 2003 November to 2004 March and those of the second season on 8 nights from 2004 December through 2005 February. The exposure times were $75-140 \mathrm{~s}$ for $B, 45-85 \mathrm{~s}$ for $V, 33-65 \mathrm{~s}$ for $R$, and $30-60 \mathrm{~s}$ for $I$, respectively, depending on weather conditions. The instrument and reduction method have been described by Lee et al. (2007) and a $2 \times 2$ binning mode was selected. The nearby stars GSC 2393-1424 and GSC 2393-1418, imaged on the chip at the same time as the variable, were selected as comparison and check stars, respectively. Coordinates and Tycho magnitudes for the three stars of interest are given in Table 1. The differential atmospheric extinction among the three stars is negligible within observational error. Measurements of the check and comparison stars indicate that the latter remained constant throughout the observing interval. The $1 \sigma$-values of the dispersions of the magnitude differences between them are about \pm 0.01 mag for all bandpasses.

A total of 2747 individual observations was obtained among the four bandpasses (711 in $B, 693$ in $V, 685$ in $R$, and 658 in $I$ ) and a sample of them is listed in Table 2. The light curves of CL Aur defined by our CCD photometry are plotted in Figure 1 as differential magnitudes versus orbital phase, which was computed according to the ephemeris for our hot-spot model determined later in this article with the Wilson-Devinney synthesis code (Wilson \& Devinney 1971, hereafter W-D). The filled and open circles are the individual measures of the first and second observing seasons, respectively. Our light curves from the first observing season had not defined secondary eclipse adequately. Except formally for the $B$ bandpass, mean brightness differences between the two seasons (in the sense of Season 1 minus Season 2) are constant and smaller than the observational error of $\pm 0.01 \mathrm{mag}:+0.013 \pm 0.014 \mathrm{mag}$ for $B,+0.007 \pm 0.016 \mathrm{mag}$ for $V,+0.001 \pm 0.017 \mathrm{mag}$ for $R$, and $-0.009 \pm 0.015 \mathrm{mag}$ for $I$, respectively. Although we show no figure illustrating variability of the color indices, their phase-locked variations are large and in the expected senses. These variations 
convey the idea of eclipses that are complete or very nearly so.

In addition, we observed several eclipse timings using a SBIG ST-8 CCD camera attached to the 35-cm reflector at the campus station of the Chungbuk National University Observatory (CbNUO) in Korea. The observations were made without a filter and reduced with the conventional IRAF package. The details of the CbNUO observations have been given by Kim et al. (2006).

\section{ORBITAL PERIOD STUDY}

We determined fifteen times of minimum light from all our CCD observations using the method of Kwee \& van Woerden (1956, hereafter KvW). These timings are listed in Table 3 together with all other CCD timings; the SOAO data are weighted means from the observations in the $B V R I$ bandpasses. The second column gives the standard deviation of each timing. For the period study of CL Aur, 198 archival timings (16 plate, 101 visual, 8 photographic and 73 CCD) have been collected from the literature and from our measurements. Most of the earlier timings were actually extracted from the data base published by Kreiner et al. (2001). Because almost all but the CCD timings were published without error information, the following standard deviations were assigned to timing residuals based on observational method: $\pm 0.022 \mathrm{~d}$ for sky-patrol plate or photographic minima, and $\pm 0.007 \mathrm{~d}$ for visual minima. Relative weights were then calculated as the inverse squares of these values consistent with the errors and weights for the CCD timings.

In order to examine whether the period change of CL Aur can be produced by a quadratic plus LTT ephemeris as suggested by W07, we fitted all times of minimum light to that ephemeris form:

$$
C=T_{0}+P E+A E^{2}+\tau_{3}
$$

where $\tau_{3}$ symbolizes the LTT effect due to a third body (Irwin 1952, 1959) and includes five parameters $\left(a_{12} \sin i_{3}, e, \omega, n\right.$ and $\left.T\right)$. Here, $n$ and $T$ denote the Keplerian mean motion of the mass center of the eclipsing pair and the epoch of its periastron passage, respectively. In this analysis, the Levenberg-Marquart technique (Press et al. 1992) was applied to solve for the eight unknown parameters of the ephemeris. By using the third-body parameters of W07 as initial values, we obtained improved (in the sense of errors smaller than formerly) parameters for them and the results are listed in Table 4 together with other related quantities. In this table, $P_{3}$ and $K$ indicate the cycle length and semi-amplitude of the LTT orbit, respectively, and $f\left(M_{3}\right)$ is the mass function of the system. Within errors, our LTT parameters are not significantly different from those of W07. The sample masses $\left(M_{3} \sin i_{3}\right)$ of the assumed third body in the table were calculated by using the absolute dimensions of the eclipsing pair presented in a later section.

The $O-C$ diagram of CL Aur constructed with the linear terms in Table 4 is drawn in the top panel of Figure 2. The timings are marked by different symbols according to observational

method and type of eclipse. The continuous curve and the dashed, parabolic one represent the 
full contribution and the quadratic term of the equation, respectively. The middle panel displays the residuals from the linear and quadratic terms, and the bottom panel the CCD residuals from the full ephemeris. These appear as $O-C_{\text {full }}$ in the fourth column of Table 3. As indicated by the figure, the quadratic plus LTT ephemeris gives a satisfactory representation of the ensemble of the residuals. In addition, another modulaton with a period of about five yrs and a semi-amplitude of about $0.0004 \mathrm{~d}$ may exist in the $O-C_{\text {full }}$ residuals. A large number of future accurate timings is required before this can be tested at an acceptable level. If the orbit of the outer component is coplanar with that of the close eclipsing pair $\left(i_{3}=88^{\circ} .2\right)$, its mass is about $M_{3}=0.83 \mathrm{M}_{\odot}$ and corresponds to a spectral type of $\mathrm{K} 1-\mathrm{K} 2$ for a normal main-sequence star. This would contribute about $0.9 \%$ to the total light of the triple system, so it will be difficult to detect such a third light source from light-curve analysis.

The quadratic term (A) of the ephemeris indicates a continuous period increase at a rate of $+(1.4 \pm 0.2) \times 10^{-7} \mathrm{~d} \mathrm{yr}^{-1}$. Because CL Aur is a semi-detached system with the less massive and cool secondary component filling its inner Roche lobe (cf. Section 4), its Roche-geometry configuration permits mass transfer from the secondary to the more massive primary star, and it is conventional

to ascribe such an increase to conservative mass transfer between the stars in the system. The calculated mass transfer rate is of the order of $1.3 \times 10^{-7} \mathrm{M}_{\odot} \mathrm{yr}^{-1}$, among the largest rates for Algol-type systems.

Alternatively, the $21.6 \mathrm{yr}$ oscillation in the $O-C$ residuals could be caused by magnetic modulation due to an activity cycle in the convective envelope of the late-type star (Applegate 1992, Lanza et al. 1998). The hot primary component of CL Aur likely has a radiative envelope as surmised from its spectral type while the less massive and cool secondary should have a shallow convective shell and at most weak magnetic activity. We applied the period $\left(P_{3}\right)$ and amplitude $(K)$ to Applegate's formulae and obtained model parameters for possible magnetic activity. The results are listed in Table 5, where the bolometric magnitude difference $\left(\Delta m_{\mathrm{rms}}\right)$ relative to the mean light level was obtained with equation (4) in the paper of Kim et al. (1997). Most of the tabulated values are close to those derived for several other binaries that appear to support Applegate's theory, but the light variation predicted from an active CL Aur secondary is at the upper limit of the theoretical value $\left(\Delta L / L_{2} \sim 0.1\right)$ proposed by him. Since our observations represent the first complete light curve, we cannot test whether such a variation has occurred in the past. However, because the secondary star is expected not to be strongly magnetically active, we think the most probable explanation of the periodic oscillation to be the LTT effect due to a low-luminosity K-type tertiary companion.

\section{LIGHT-CURVE SYNTHESIS}

As shown in Figure 1, our observations clearly indicate that the light curve morphology of CL Aur is not $\beta$ Lyr type but rather very similar to that of Algol, its class prototype. To understand the geometrical structure and the physical parameters of the system, our $B V R I$ light curves were 
solved simultaneously in a manner similar to those for XX Cep (Lee et al. 2007) and GW Gem (Lee et al. 2009a) by using the latest version 2 of the W-D code and an extensive $q$-search procedure. The surface temperature of the primary star was fixed at $T_{1}=9,420 \mathrm{~K}$, according to its spectral type A0 and Harmanec's (1988) table. We had attempted to improve the spectral classification by obtaining high-resolution spectra with the echelle spectrograph attached to the 1.8-m telescope at Bohyunsan Optical Astronomical Observatory (BOAO) in Korea (Kim et al. 2002). These images were not well enough exposed to improve upon the literature value but indicated a similar result. Initial bolometric and monochromatic limb-darkening coefficients were taken from the tables of van Hamme (1993) and were used together with the model atmosphere option. The $q$-search for modes 2, 3, 4 and 5 of the synthesizing code (Wilson \& Biermann 1976) converged and showed acceptable photometric solutions only for mode 5 (semi-detached systems for which the secondary stars fill the inner Roche lobes). As displayed in Figure 3, the optimal solution is around $q=0.60$. This undertanding conforms to the sense of the mass transfer from the secondary component to the hotter, more massive primary star suggested by the period study.

The $q$ value was treated as an adjustable parameter in all subsequent syntheses deriving binary parameters. The best result for unspotted photospheres is listed in columns (2) and (3) of Table 6 and the residuals from the analysis are plotted in the left panels of Figure 4, where phase-locked, unmodelled light variations are indicated. Such features can be reasonably attributed to a hot spot on the surface of the primary as a result of the impact of the gas stream from the cooler, less massive secondary star. This situation is known for the Algol-type, semi-detached binary RZ Cas (Rodriguez et al. 2004).

This interpretation does not exclude the possible existence of magnetic cool spots located on the surface of the late-type star. We therefore tested two spot models: a hot spot on the more massive primary star due to mass transfer and a cool spot on the secondary star caused by magnetic activity. In a formal sense, as shown by the entries on the last line of Table 6 , the hot spot model does improve the light-curve fit. Separate trials for a cool spot on the secondary star were not so successful as for the hot-spot model. Final results for all the light curves are given in columns (4) and (5) of Table 6 and the residuals from our hot-spot model are shown in the right panels of Figure 4. As seen in the figure, there is a slight improvement in the residuals for the spot model at about phase 0.87 in all panels compared to the unspotted model. This orbital phase is almost exactly where we would expect the effects of a gas stream to be evident. The hot spot agrees well with the concept of mass transfer from the secondary to the primary component inferred from the period analysis and from the relatively large size of the hot star. In addition, there are small systematic differences between the two seasons for the light residuals, which means that mass transfer activity may be variable, as is sometimes reported for light curves of Algol binaries. In all these trials, a possible third light source $\left(\ell_{3}\right)$ was considered but the results remained indistinguishable from 0.00 within their errors. We fixed $\ell_{3}$ to be 0.0 during the final light-curve analysis.

\footnotetext{
${ }^{2} \mathrm{ftp}$ ://ftp.astro.ufl.edu/pub/wilson/
} 
Because of the almost complete eclipses, the light curve determinacy for CL Aur is quite high. In idealized Roche geometry, the impact of the gas stream should be on the primary star's equator but this is not the case for CL Aur. We return to this detail later. It could also be true that both hot and cool spots exist and that our data are sufficient to isolate only the dominant one.

A recent study by Lee et al. (2009b) indicates that the minimum epochs of the cool contact binary AR Boo have been systematically shifted by light asymmetries due to spot activity, as predicted by Maceroni \& van't Veer (1994). To check this possibility for this Algol-type system, we calculated the timings for each of our CL Aur eclipses with the W-D code. The results are listed in the second column of Table 7, together with the minimum times obtained by the KvW method for comparison. We can see that the differences among them in column (4) of Table 7 are within the precision of each $\mathrm{KvW}$ minimum and the hot spot does not inflect those timings. This agreement is doubtless due to the small temperature contrast of the spot against the photosphere and the small angular deviation of the spot from the systemic line of centers.

A new class of $\delta$ Sct stars designated as oEA (oscillating EA) objects (Mkrtichian et al. 2004) has been identified as the (B)A-F spectral type mass-gaining components of Algol-type semidetached systems. About half of them have been discovered through a photometric survey project run by a Korean group (cf. Kim et al. 2003) in order to search for A-type pulsating components in classical Algols (Soydugan et al. 2006). The oEA stars have pulsational amplitudes and periods similar to classical $\delta$ Sct stars but a different evolutionary scenario due to mass accretion. From its spectral type, the primary star of CL Aur would be a candidate for such pulsations. Therefore, we applied the discrete fourier transform program PERIOD04 (Lenz \& Breger 2005) to the light curve residuals from our binary models, but there was found no periodicity with a semi-amplitude larger than 3 mmag.

\section{ESTIMATED ABSOLUTE DIMENSIONS}

The foregoing presents a consistent picture of CL Aur in the sense that a determinate representation of the light curve has been achieved and that the secular period variability has a highly probable cause consistent with the light curve interpretation. Now it is necessary to look at further consequences of this description.

Absolute dimensions of CL Aur can be estimated from our photometric solutions with the hot-spot model in Table 6 and Harmanec's relation between spectral type and mass. By assuming the primary star to be a normal main-sequence one with a spectral type of $\mathrm{A} 0 \mathrm{~V}$, the astrophysical parameters for the components were obtained to be those listed in Table 8, where the radii are the mean volume radii calculated from the tables of Mochnacki (1984). The luminosities and

the bolometric magnitudes were computed by adopting $T_{\text {eff } \odot}=5,780 \mathrm{~K}$ and $M_{\text {bol } \odot}=+4.69$. The bolometric corrections were obtained from the relation between $\log T$ and $\mathrm{BC}$ given by Kang et al. (2007). The intrinsic color of $(B-V)_{0}=+0.06$ for the binary system was estimated from their 
calibration $(B-V)_{1}$ versus $T_{1}$ of the primary star and from our computed light ratio; it leads to $E(B-V)=+0.28$. For lack of better evidence, it is prudent to assume that the mean system color index and interstellar reddening can be in error by as much as \pm 0.05 . With this qualification and with the values of $V$ and $M_{\mathrm{V} \text {,total }}$ and the interstellar extinction of $A_{\mathrm{V}}=3.1 E(B-V)$, we have calculated a nominal distance to the system of about 1,150 pc and an accidental error associated this distance of about $5 \%$. This result is also misleading for another reason: one does not know the phase at which the Tycho measures were made so there is no assurance that they refer to maximum light. The total error on the distance determination may well be $20 \%$.

From the estimated parameters of CL Aur, it is possible to consider the evolutionary state of the eclipsing system in mass-radius and mass-luminosity diagrams. The primary star lies in the main-sequence band between the ZAMS and the TAMS, while the secondary is larger and brighter than expected for its mass. The locations of the components in the Hertzsprung-Russell (HR) diagram are shown in Figure 5, together with single-star evolutionary tracks having masses of 2.20 $M_{\odot}$ and $1.35 M_{\odot}$ and solar metallicity (Girardi et al. 2000). There also appear in the figure theoretical mass-conserving evolutionary tracks for model donor (3.0 $M_{\odot}$, the present secondary) and gainer $\left(1.2 M_{\odot}\right)$ stars belonging to a system with a total mass of $4.2 M_{\odot}$ and an initial orbital period of 1.0 d calculated by the Brussels group 3 (De Loore \& van Rensbergen, 2005). Although these tracks are not for a system with the same mass ratio and total mass as CL Aur $\left(3.6 M_{\odot}\right)$, the two components can be scaled to fit the concept of a similar conservative model quite well, indicating that the binary system may still be undergoing mass transfer as a result of case A evolution. The present age of the system is estimated to be about 0.24 Gyr.

\section{DISCUSSION AND CONCLUSIONS}

We next probed the credibility of the hot (inferentially an impact) spot model motivated largely by two recognitions: (a) the two arrays of light curve residuals in Figure 4 do not appear very different one from another although formally the light curve fitting is improved by the assumption of a spot and (b) the center of the spot is sensibly distant from the stellar equator and orbital plane.

The first matter is the consequence of the envelope of the residuals responding weakly to the spot modeling although the residuals of small absolute value did respond well with considerably reduced values on average. This can only be construed as a situation in which there exist residuals of noise much greater than most of the rest of them. In principle, these residuals could be produced by a combination of physical causes such as magnetic activity from the cool secondary and a change in the mass transfer rate. However, the actual noise level is not significantly larger than that $( \pm 0.01$ mag) of SOAO data made during the last few observing seasons and probably can be traced to

\footnotetext{
${ }^{3}$ http://we.vub.ac.be/astrofys/
} 
marginal observing conditions on some nights.

The location of the spot has more physical interest. First, an extensive set of restricted 3body calculations examined the longitude coordinate of the spot on the hot primary for a range $\left(0.0000003 \mathrm{~km} \mathrm{~s}^{-1}-450 \mathrm{~km} \mathrm{~s}^{-1}\right)$ of initial velocities streaming from the $\mathrm{L}_{1}$ point as shown in the first column of Table 9 . This range obviously includes the thermal velocity in the cool star envelope with the lower limit of the range essentially that of free-fall. For the greatest extent of this velocity range the impact spot is close (within $21^{\circ}$ ) to the line of centers. In the hydrodynamic computations of Lubow \& Shu (1975), just such an effect appears with the streaming gas deflected about $20^{\circ}$ from the line of centers, not nearly enough to avoid contact with the large, detached primary. From this point of view, the credibility of the concept of impact is therefore high, and conservative mass transfer of the streaming material must be considered likely. These calculations also make it difficult to imagine any stable accretion structures around the hot star but they invariably led to an orbital-plane spot rather than the modeled one of co-latitude very different from $90^{\circ}$. Another suite of calculations, also given in Table 9, showed the expected result: inital non-zero-velocity $z$-components in the streaming gas led to impacts away from the hot stellar equator and these did not have to be large in order to fall $15-20^{\circ}$ away from the equator. Is this result to be taken as evidence that the spot really exists at its modeled location or is it just a dynamical truism and doesn't verify the spot existence at all? The resolution of this quandary requires a mechanism to move the gas appropriately and three possible ones come to mind. (a) Turbulence in the gas moving from the $\mathrm{L}_{1}$-point caused a concentration of it to fall at the modeled position during the two observing seasons. (b) A weak magnetic field seated in the cool star had at least one component channeling the ionized fraction of the streaming gas to the modeled impact spot. (c) The solution for the spot is possibly not so unique as W-D indicates. For want of independent evidence, each of these is an unprovable hypothesis.

More information does exist, however. We calculated the impact luminosity from the stream at the rate of mass transfer given by the interpretation of the $O-C$ diagram. For the same range (and in the same sense as above) of stream velocity, the impact luminosity varied between $3.2 L_{\odot}$ and $0.7 L_{\odot}$. If the impact energy is partitioned between virialization into the gainer star and spot luminosity as Hilditch (1989) proposes, the least luminous output from a spot should therefore be about $0.35 L_{\odot}$. This is to be compared to the spot luminosity from its black body W-D model of about $4 \times 10^{-5} L_{\odot}$. The magnitude of the discrepancy means either (a) that conversion from kinetic to luminous energy is very inefficient or (b) that the mass-motions rate indicated by the $O-C$ diagram is not restricted to material leaving the $\mathrm{L}_{1}$ point or (c) some combination of these two ideas or (d) that the concept of a spot is itself incorrect. For (a) to be a realistic interpretation, deep penetration of the impacting gas into the hot star is required so that most of the impacting gas becomes thermalized in the hot star. It is also necessary to postulate an envelope circulation pattern that re-surfaces at the modeled spot position for the residual gas that has not been thermalized. If (b) is to be entertained seriously, there must be substantial mass lost by evaporation from the entire Roche lobe that is the photosphere of the cool star. This demands only a small $\left(\sim 10^{-4}\right)$ 
contribution to the $O-C$ diagram from the gas leaving $\mathrm{L}_{1}$ with the majority of the mass and angular momentum loss contributed by that departing the rest of the cool star's surface. On a small scale the transfer would be conservative as usual but large scale mass loss would be the dominant cause of the $A$-term in the ephemeris. Hypothesis (d) would be the most economical interpretation but conflicts with the impersonal syntheses of the light curves. At this time, we favor a combination of interpretations (a) and (b).

In summary, our study of the orbital period and the light curves reveals that CL Aur is a classical Algol-type interacting system with the less massive and cool secondary star filling its inner Roche lobe. The possibility of a hot-spot model due to impact of streaming gas onto the hot star has led to confusing difficulties that we have resolved only tentatively. High-resolution, near-IR spectroscopy should reveal the lines of the secondary star and lead to accurate absolute parameters to replace our estimates. Moreover, there is also the possibility of obtaining direct evidence of mass-transfer activity, such as complex and variable line profiles of $\mathrm{H} \alpha$ or variations in the strength of the O I 7774 absorption line. The evolutionary status of the system will then be more convincingly in hand.

The more general reality of things is that there are many short-period Algols which have not been studied so deeply as this present work has examined CL Aur. It would make a significant advance if more of these - having different algebraic signs for the secular term of the period variability - could be brought to the same level of knowledge as the present binary. For instance, should all such binaries with $A>0$ require a spot on the hot star near the line of centers, there would be major support for the reasoning concerning mass movements. Should it also happen that the spot luminosity was consistently found to be lower than the kinetic impact required for $50 \%$ energy conversion, there would be good reason to believe that the majority of the mass lost from the cool secondaries is, in fact, lost to the systems and not transferred conservatively. The short-period binaries found to require mode 4 (semi-detached systems with the primary stars filling its inner Roche lobes) for their representations would be expected, then, to show small-scale mass transfer to the secondaries and systemic mass loss from the primaries. Much valuable observational and modeling work remains.

We would like to thank the staff of the Chungbuk National University Observatory for assistance with our observations, and Mr. Jae-Rim Koo for the spectroscopic observations of CL Aur. We appreciate the careful reading and valuable comments of the anonymous referee. We have used the Simbad data base maintained at CDS many times and appreciate its availability. This work has been done as part of a cooperative project between Chungbuk National University and the Korea Astronomy and Space Science Institute. C.-H. K. was supported by the Korea Research Foundation (KRF) Grant funded by the Korea government (MEST) (No. 2009-0069330). 


\section{REFERENCES}

Agerer, F., \& Hübscher, J. 2002, Inf. Bull. Variable Stars, No. 5296

Applegate, J. H. 1992, ApJ, 385, 621

Baldwin, M. E., \& Samolyk, G. 2004, Observed Minima Timings of Eclipsing Binaries, No. 9 (Cambridge: AAVSO)

Baldwin, M. E., \& Samolyk, G. 2007, Observed Minima Timings of Eclipsing Binaries, No. 12 (Cambridge: AAVSO)

Bíró, I. B., et al. 2007, Inf. Bull. Variable Stars, No. 5753

Blättler, E. 2000, BBSAG Bull., No. 121

Borkovits, T., et al. 2008, Inf. Bull. Variable Stars, No. 5835

Brát, L., Zejda, M., \& Svoboda, P. 2007, Open Eur. J. Var. Stars, 74, 1

Brát, L., et al. 2008, Open Eur. J. Var. Stars, 94, 1

Brát, L., et al. 2009, Open Eur. J. Var. Stars, 107, 1

De Loore, C., \& van Rensbergen, W. 2005, Ap\&SS, 296, 353

Diethelm, R. 2001, BBSAG Bull., No. 126

Doğru, S. S., Dönmez, A., Tüysüz, M., Doğru, D., Özkardeş, B., Soydugan, E., \& Soydugan, F. 2007, Inf. Bull. Variable Stars, No. 5746

ESA. 1997, The Hipparcos and Tycho Catalogues (ESA SP-1200; Noordwijk: ESA)

Girardi, L., Bressan, A., Bertelli, G., \& Chiosi, C. 2000, A\&AS, 141, 371

Götz, W., \& Wenzel, W. 1968, Mitteilungen Veränderliche Sterne, 5, 5

Hall, D. S. 1989, Space Sci. Rev., 50, 219

Harmanec, P. 1988, Bull. Astron. Inst. Czechoslovakia, 39, 329

Hegedüs, T. 1988, Bull. Inf. CDS, 35, 15

Hilditch, R. W. 1989, Space Sci. Rev., 50, 289

Hoffleit, D. 1935, Harvard Bulletin, 901, 20

Høg, E., et al. 2000, A\&A, 355, 27

Hübscher, J. 2007, Inf. Bull. Variable Stars, No. 5802 
Hübscher, J., Paschke, A., \& Walter, F. 2005, Inf. Bull. Variable Stars, No. 5657

Hübscher, J., Paschke, A., \& Walter, F. 2006, Inf. Bull. Variable Stars, No. 5731

Hübscher, J., \& Walter, F. 2007, Inf. Bull. Variable Stars, No. 5761

Irwin, J. B. 1952, ApJ, 116, 211

Irwin, J. B. 1959, AJ, 64, 149

Kang, Y.-W., Hong, K.-S., \& Lee, J. 2007, in ASP Conf. Ser. 362, The Seventh Pacific Rim Conference on Stellar Astrophysics, ed. Y.-W. Kang et al. (San Francisco: ASP), 19

Kim, C.-H., Jeong, J. H., Demircan, O., Müyesseroğlu, Z., \& Budding, E. 1997, AJ, 114, 2753

Kim, C.-H., Lee, C.-U., Yoon, Y.-N., Park, S.-S., Kim, D.-H., Cha, S.-M., \& Won, J.-H. 2006, Inf. Bull. Variable Stars, No. 5694

Kim, S.-L., Lee, J. W., Kwon, S.-G., Youn, J.-H., Mkrtichian, D. E., Kim, C. 2003, A\&A, 405, 231

Kim, K.-M. et al., 2002, J. Korea Astron. Soc., 35, 221

Kreiner, J. M., Kim, C.-H., \& Nha, I.-S. 2001, An Atlas of $O-C$ Diagrams of Eclipsing Binary Stars (Krakow: Wydawn. Nauk. Akad. Pedagogicznej)

Kurochkin, N. E. 1951, Variable Stars, 8, 351

Kwee, K. K., \& van Woerden, H. 1956, Bull. Astron. Inst. Netherlands, 12, 327

Lanza, A. F., Rodono, M., \& Rosner, R. 1998, MNRAS, 296, 893

Lee, J. W., Kim, C.-H., \& Koch, R. H. 2007, MNRAS, 379, 1665

Lee, J. W., Kim, S.-L., Lee, C.-U., Kim, H.-I., Park, J.-H., Park, S.-R., \& Koch, R. H. 2009a, PASP, 121, 104

Lee, J. W., Youn, J.-H., Lee, C.-U., Kim, S.-L., \& Koch, R. H. 2009b, AJ, 138, 478

Lenz, P., \& Breger, M. 2005, Comm. Asteroseismology, 146, 53

Lubow, S. H., \& Shu, F. H. 1975, ApJ, 198, 383

Maceroni, C., \& van't Veer, F. 1994, A\&A, 289, 871

Mkrtichian, D. E., et al. 2004, A\&A, 419, 1015

Mochnacki, S. W. 1984, ApJS, 55, 551

Nelson, R. H. 2006, Inf. Bull. Variable Stars, No. 5672 
Press, W. H., Teukolsky, S. A., Vetterling, W. T., \& Flannery, B. P. 1992, Numerical Recipes (Cambridge: Cambridge Univ. Press), Chapter 15

Richards, M. T., \& Albright, G. E. 1999, ApJS, 123, 537

Rodriguez, E., et al. 2004, MNRAS, 347, 1317

Smith, A. B., \& Caton, D. B. 2007, Inf. Bull. Variable Stars, No. 5745

Soydugan, E., Soydugan, F., Demircan, O., \& İbanoglu, C. 2006, MNRAS, 370, 2013

Van Hamme, W. 1993, AJ, 106, 209

Wilson, R. E., \& Biermann, P. 1976, A\&A, 48, 349

Wilson, R. E., \& Devinney, E. J. 1971, ApJ, 166, 605

Wolf, M., S̆arounová, L., Brož, M., \& Horan, R. 1999, Inf. Bull. Variable Stars, No. 4683

Wolf, M., et al. 2007, Inf. Bull. Variable Stars, No. 5780 (W07)

This preprint was prepared with the AAS IATEX macros v5.2. 


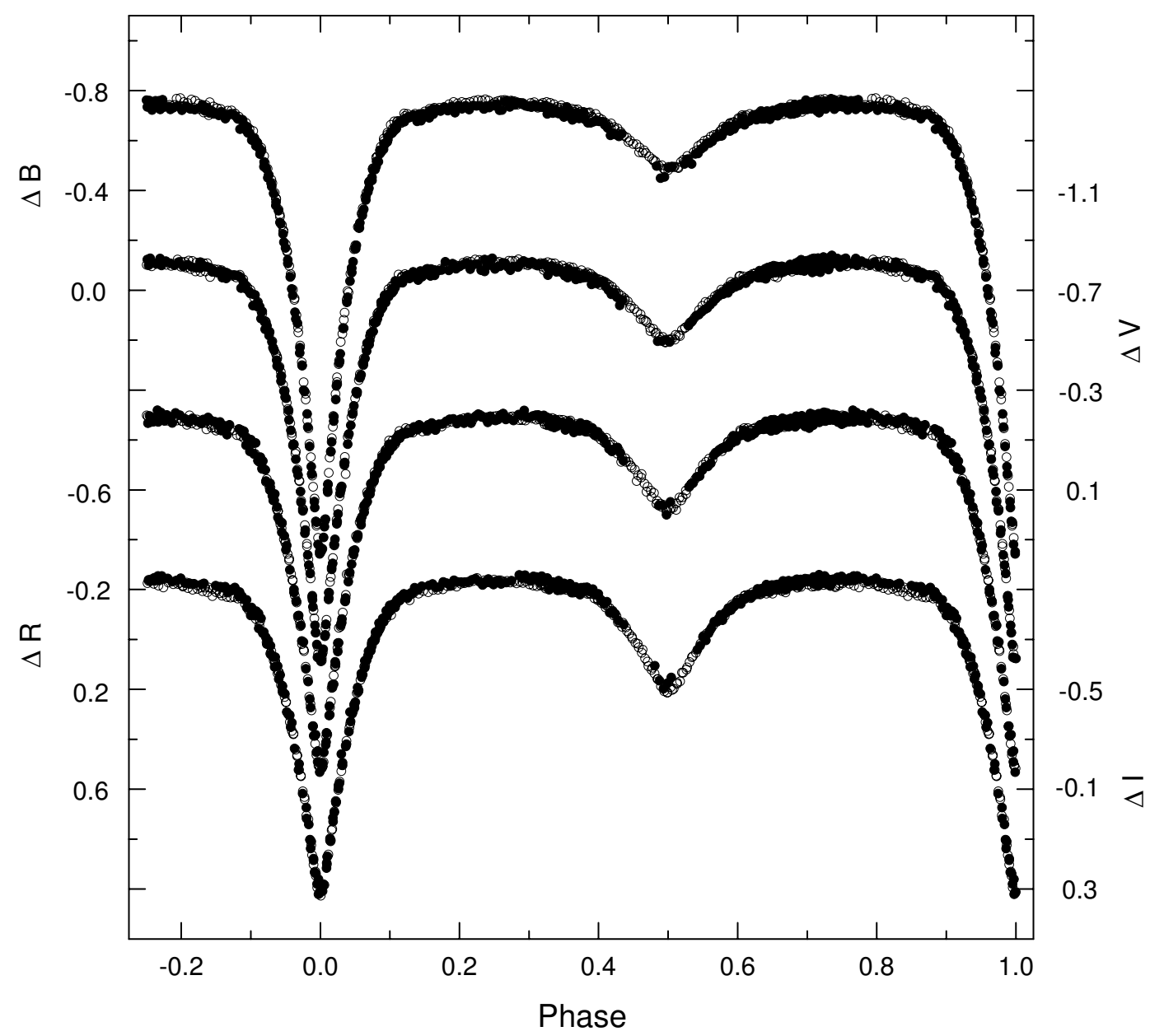

Fig. 1.- SOAO light curves of CL Aur in the $B, V, R$, and $I$ bandpasses. The filled and open circles are the individual measures of the first (2003 Nov-2004 Mar) and second (2004 Dec-2005 Feb) seasons, respectively. Because of the high density of the points, many of the 2004 measures cannot be seen individually. 


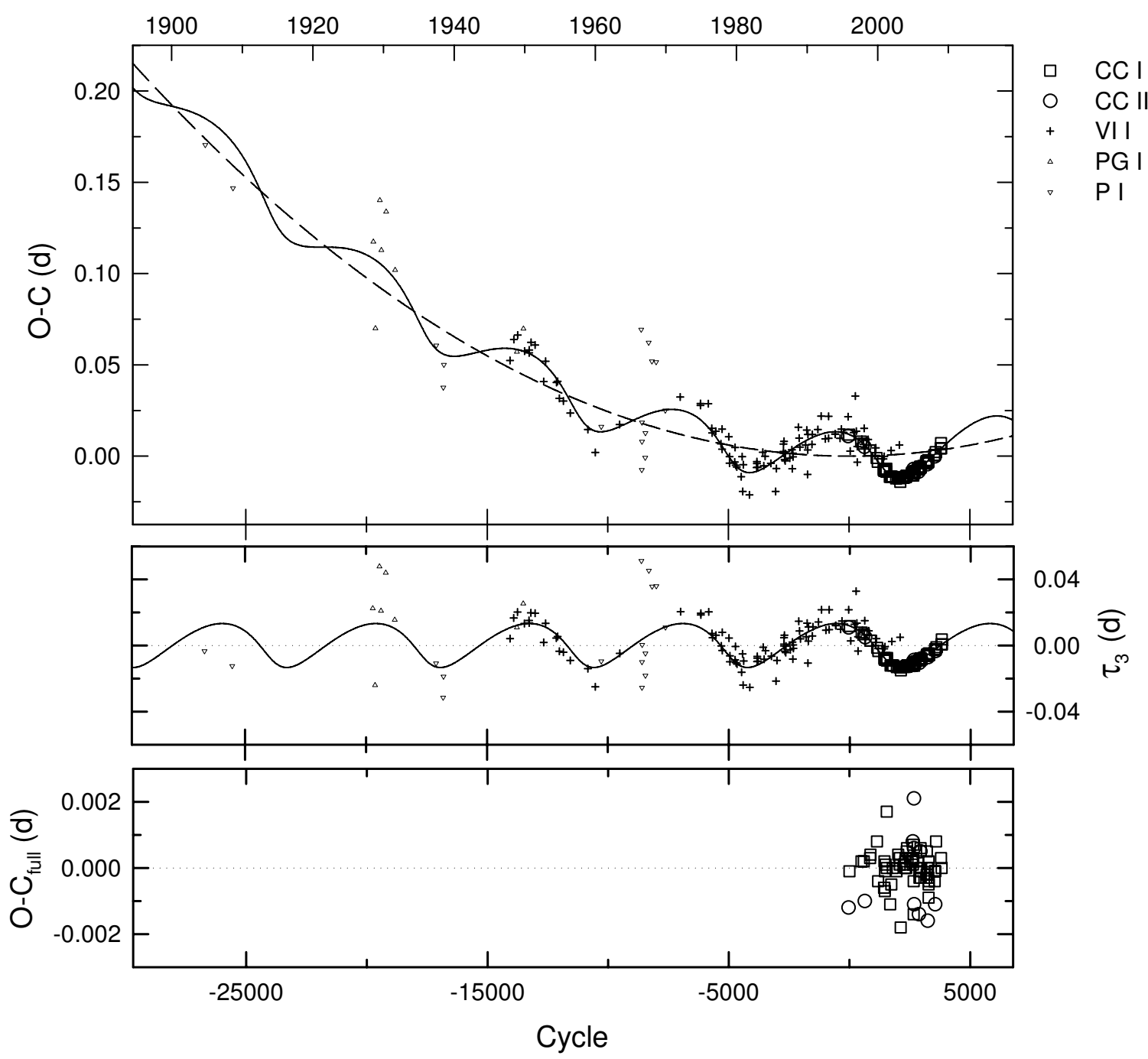

Fig. 2.- The $O-C$ diagram of CL Aur constructed with the linear terms in Table 4. In the top panel, the continuous curve and the dashed, parabolic one represent the full contribution and the quadratic term of the equation, respectively. The middle panel displays the residuals from the linear and quadratic terms, and the bottom panel the CCD residuals from the complete ephemeris. CC, VI, PG, and P denote CCD, visual, photographic, and photographic plate minima, respectively. 


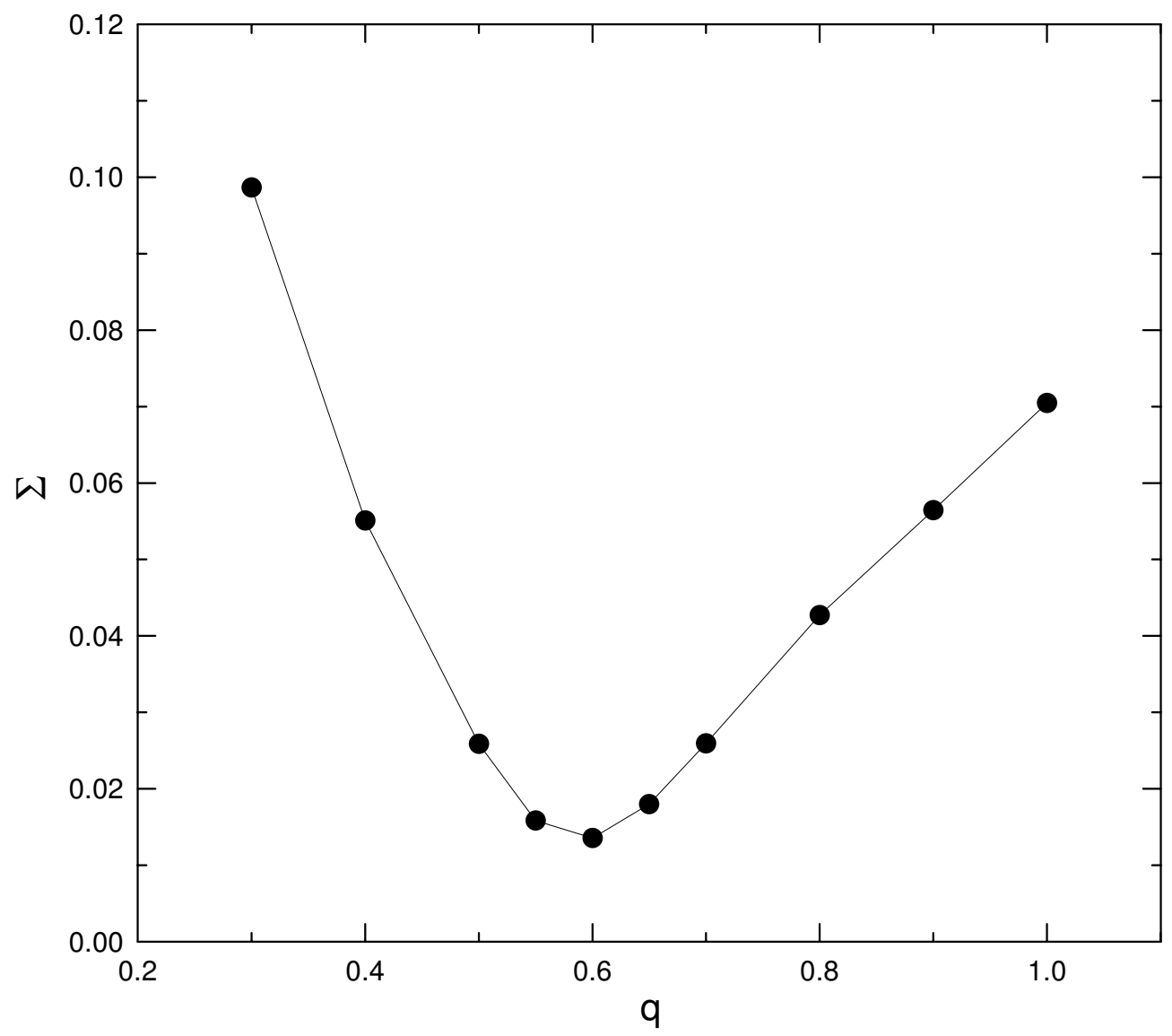

Fig. 3.- The behavior of $\Sigma$ (the sum of the residuals squared) of CL Aur as a function of mass ratio $q$, showing a minimum value near $q=0.60$ for Mode 5 of the $\mathrm{W}$-D code. 


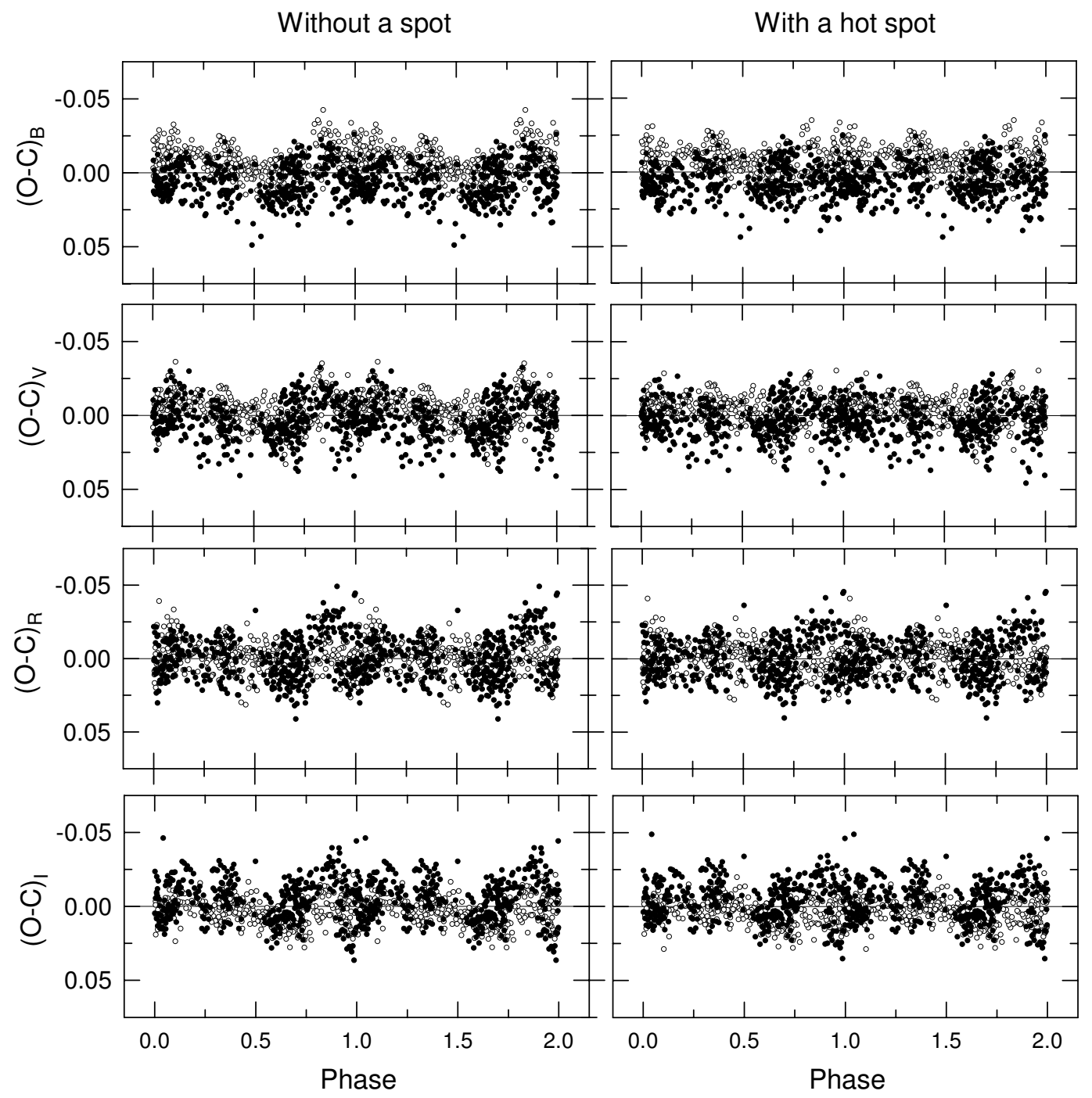

Fig. 4. - The magnitude residuals in $B, V, R$ and $I$ corresponding to two binary models in columns (2)-(5) of Table 6: without (left panels) and with (right panels) a hot spot on the primary component. The symbols are plotted in the same sense as Figure 1. 


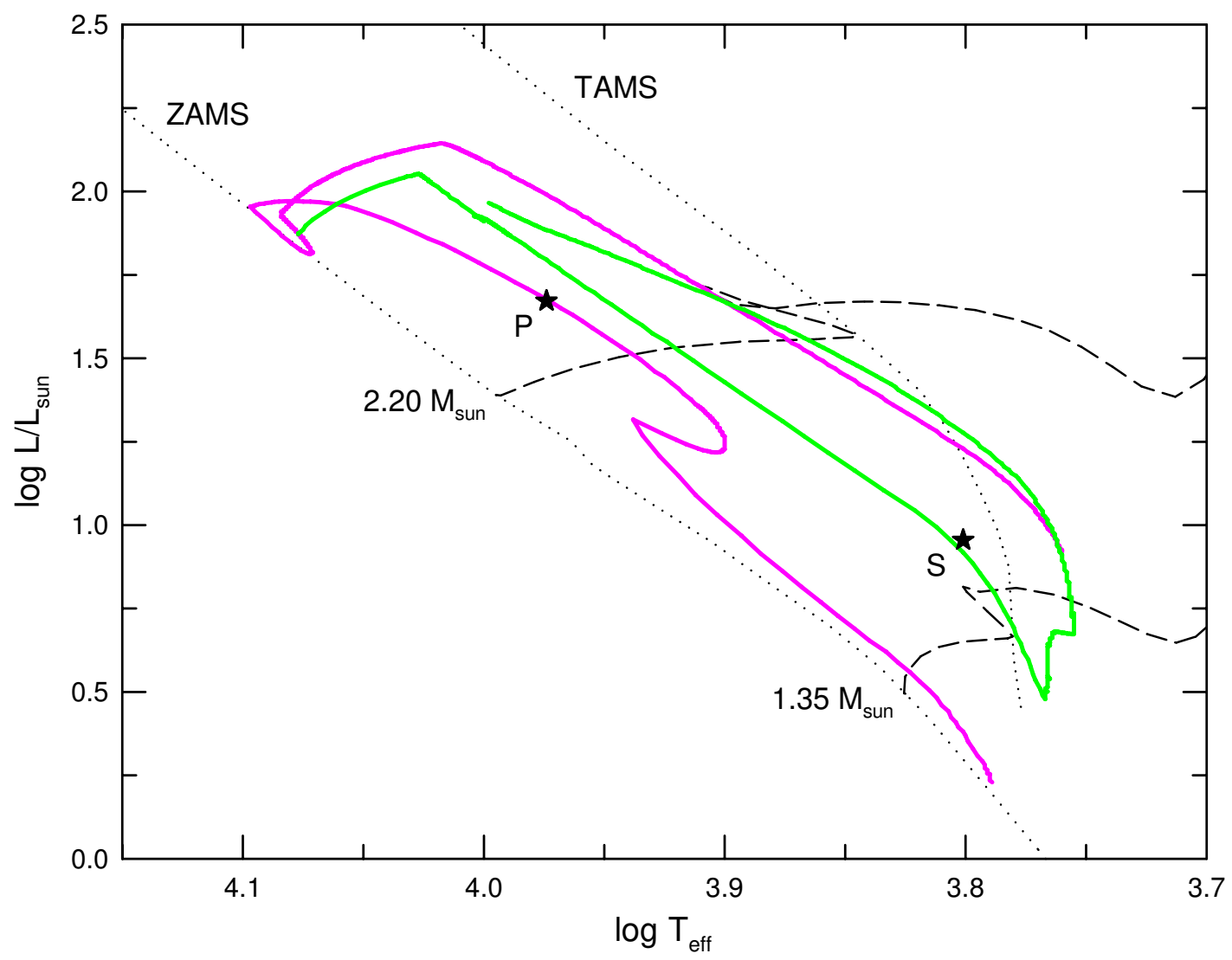

Fig. 5.- The HR diagram of the primary (P) and secondary (S) stars of CL Aur. The dashed lines correspond to single star evolutionary tracks for the present masses of both components. The green- and pink-colored lines represent theoretical tracks for closely similar donor and gainer stars, respectively. See the text for details. 
Table 1. Coordinates and photometric data for the program stars.

\begin{tabular}{cccccc}
\hline \hline Star & GSC & RA $(J 2000)$ & DEC $(J 2000)$ & $V_{\mathrm{T}}^{\dagger}$ & $(B-V)_{\mathrm{T}}^{\dagger}$ \\
\hline CL Aurigae & $2393-1455$ & $05^{\mathrm{h}} 12^{\mathrm{m}} 54.19$ & $+33^{\circ} 30^{\prime} 28^{\prime \prime} .4$ & +11.65 & +0.39 \\
Comparison & $2393-1424$ & $05^{\mathrm{h}} 13^{\mathrm{m}} 27^{\mathrm{s}} 48$ & $+33^{\circ} 26^{\prime} 46^{\prime \prime} \cdot 3$ & - & - \\
Check & $2393-1418$ & $05^{\mathrm{h}} 12^{\mathrm{m}} 19^{\mathrm{s}} .08$ & $+33^{\circ} 26^{\prime} 31^{\prime \prime} .5$ & +12.30 & +0.14 \\
\hline
\end{tabular}

${ }^{\dagger}$ From the Tycho-2 Catalogue (Høg et al. 2000).

Table 2. SOAO CCD photometric observations of CL Aur.

\begin{tabular}{cccccccc}
\hline \hline HJD & $\Delta B$ & HJD & $\Delta V$ & HJD & $\Delta R$ & HJD & $\Delta I$ \\
\hline $2,452,974.23806$ & 0.707 & $2,452,974.23966$ & 0.525 & $2,452,974.24094$ & 0.348 & $2,452,974.23583$ & 0.104 \\
$2,452,974.24426$ & 0.872 & $2,452,974.24587$ & 0.673 & $2,452,974.24716$ & 0.452 & $2,452,974.24215$ & 0.179 \\
$2,452,974.24997$ & 1.020 & $2,452,974.25159$ & 0.775 & $2,452,974.25288$ & 0.532 & $2,452,974.24832$ & 0.280 \\
$2,452,974.25568$ & 1.042 & $2,452,974.25732$ & 0.760 & $2,452,974.25861$ & 0.496 & $2,452,974.25404$ & 0.312 \\
$2,452,974.26142$ & 0.949 & $2,452,974.26304$ & 0.682 & $2,452,974.26433$ & 0.412 & $2,452,974.25977$ & 0.281 \\
$2,452,974.26721$ & 0.800 & $2,452,974.26882$ & 0.540 & $2,452,974.27010$ & 0.305 & $2,452,974.26550$ & 0.196 \\
$2,452,974.27288$ & 0.646 & $2,452,974.27450$ & 0.420 & $2,452,974.27579$ & 0.193 & $2,452,974.27127$ & 0.106 \\
$2,452,974.27860$ & 0.507 & $2,452,974.28020$ & 0.299 & $2,452,974.28149$ & 0.098 & $2,452,974.27697$ & 0.030 \\
$2,452,974.28423$ & 0.377 & $2,452,974.28585$ & 0.198 & $2,452,974.28714$ & 0.014 & $2,452,974.28266$ & -0.049 \\
$2,452,974.29000$ & 0.255 & $2,452,974.29161$ & 0.088 & $2,452,974.29289$ & -0.082 & $2,452,974.28831$ & -0.123 \\
\hline
\end{tabular}

Note. - This table is available in its entirety in machine-readable and Virtual Observatory (VO) forms in the online journal and also at the Web page (http://binary.cbnu.ac.kr/bbs/zboard.php?id=lab_photometry). A portion is shown here for guidance regarding its form and content. 
Table 3. Observed CCD times of minimum light for CL Aur.

\begin{tabular}{|c|c|c|c|c|c|}
\hline HJD & Error & Epoch & $O-C_{\text {full }}$ & Min & References \\
\hline $2,450,044.3958$ & \pm 0.0007 & -42.5 & -0.00122 & II & Wolf et al. (1999) \\
\hline $2,450,097.2826$ & \pm 0.0002 & 0.0 & -0.00014 & $\mathrm{I}$ & Wolf et al. (1999) \\
\hline $2,450,714.4888$ & \pm 0.0006 & 496.0 & 0.00020 & $\mathrm{I}$ & Wolf et al. (1999) \\
\hline $2,450,831.4589$ & \pm 0.0001 & 590.0 & 0.00019 & $\mathrm{I}$ & Wolf et al. (1999) \\
\hline $2,450,884.3431$ & \pm 0.0003 & 632.5 & -0.00100 & II & Wolf et al. (1999) \\
\hline $2,451,157.4815$ & \pm 0.0006 & 852.0 & 0.00027 & $\mathrm{I}$ & Wolf et al. (1999) \\
\hline $2,451,177.3914$ & \pm 0.0001 & 868.0 & 0.00043 & $\mathrm{I}$ & Wolf et al. (1999) \\
\hline $2,451,508.391$ & \pm 0.0030 & 1134.0 & 0.00080 & $\mathrm{I}$ & Blättler (2000) \\
\hline $2,451,570.6077$ & \pm 0.0003 & 1184.0 & -0.00037 & $\mathrm{I}$ & Baldwin \& Samolyk(2004) \\
\hline $2,451,880.4526$ & \pm 0.0002 & 1433.0 & -0.00061 & $\mathrm{I}$ & Brát et al. (2009) \\
\hline $2,451,901.6067$ & \pm 0.0002 & 1450.0 & -0.00062 & $\mathrm{I}$ & Wolf et al. (2007) \\
\hline $2,451,901.6075$ & \pm 0.0003 & 1450.0 & 0.00018 & $\mathrm{I}$ & Brát et al. (2009) \\
\hline $2,451,921.5164$ & \pm 0.0002 & 1466.0 & -0.00068 & $\mathrm{I}$ & Agerer \& Hübscher (2002) \\
\hline $2,451,925.2501$ & \pm 0.0021 & 1469.0 & -0.00006 & $\mathrm{I}$ & Brát et al. (2007) \\
\hline $2,451,956.3593$ & \pm 0.0015 & 1494.0 & 0.00014 & $\mathrm{I}$ & Brát et al. (2007) \\
\hline $2,452,013.5998$ & \pm 0.0002 & 1540.0 & 0.00004 & $\mathrm{I}$ & Baldwin \& Samolyk(2004) \\
\hline $2,452,017.3345$ & \pm 0.0003 & 1543.0 & 0.00165 & $\mathrm{I}$ & Wolf et al. (2007) \\
\hline $2,452,196.5200$ & \pm 0.0003 & 1687.0 & -0.00113 & $\mathrm{I}$ & Diethelm (2001) \\
\hline $2,452,252.5171$ & \pm 0.0001 & 1732.0 & -0.00047 & $\mathrm{I}$ & Wolf et al. (2007) \\
\hline $2,452,333.4014$ & \pm 0.0001 & 1797.0 & -0.00002 & $\mathrm{I}$ & Wolf et al. (2007) \\
\hline $2,452,522.5455$ & \pm 0.0001 & 1949.0 & -0.00013 & $\mathrm{I}$ & Wolf et al. (2007) \\
\hline $2,452,609.6521$ & \pm 0.0001 & 2019.0 & 0.00038 & $\mathrm{I}$ & Baldwin \& Samolyk(2004) \\
\hline $2,452,684.3143$ & \pm 0.0003 & 2079.0 & 0.00012 & $\mathrm{I}$ & Wolf et al. (2007) \\
\hline $2,452,690.5364$ & \pm 0.0002 & 2084.0 & 0.00034 & $\mathrm{I}$ & Baldwin \& Samolyk(2004) \\
\hline $2,452,731.5986$ & \pm 0.0007 & 2117.0 & -0.00185 & $\mathrm{I}$ & Baldwin \& Samolyk(2004) \\
\hline $2,452,899.5915$ & \pm 0.0002 & 2252.0 & 0.00012 & $\mathrm{I}$ & Wolf et al. (2007) \\
\hline $2,452,964.2991$ & \pm 0.0001 & 2304.0 & 0.00001 & $\mathrm{I}$ & Wolf et al. (2007) \\
\hline $2,452,974.2543$ & \pm 0.0002 & 2312.0 & 0.00017 & $\mathrm{I}$ & This paper (SOAO) \\
\hline $2,452,986.6982$ & \pm 0.0002 & 2322.0 & 0.00028 & $\mathrm{I}$ & Baldwin \& Samolyk(2004) \\
\hline $2,453,019.0518$ & \pm 0.0001 & 2348.0 & 0.00000 & $\mathrm{I}$ & This paper (SOAO) \\
\hline $2,453,055.1393$ & \pm 0.0002 & 2377.0 & 0.00046 & $\mathrm{I}$ & This paper (SOAO) \\
\hline $2,453,062.6057$ & \pm 0.0002 & 2383.0 & 0.00058 & $\mathrm{I}$ & Baldwin \& Samolyk(2004) \\
\hline $2,453,323.9256$ & \pm 0.0001 & 2593.0 & 0.00022 & $\mathrm{I}$ & Baldwin \& Samolyk (2007) \\
\hline
\end{tabular}


Table 3-Continued

\begin{tabular}{|c|c|c|c|c|c|}
\hline HJD & Error & Epoch & $O-C_{\text {full }}$ & Min & References \\
\hline $2,453,350.0577$ & \pm 0.0001 & 2614.0 & 0.00027 & $\mathrm{I}$ & This paper (SOAO) \\
\hline $2,453,353.1692$ & \pm 0.0006 & 2616.5 & 0.00081 & II & This paper (SOAO) \\
\hline $2,453,360.0128$ & \pm 0.0001 & 2622.0 & 0.00030 & I & This paper (SOAO) \\
\hline $2,453,361.2570$ & \pm 0.0002 & 2623.0 & 0.00011 & I & This paper (SOAO) \\
\hline $2,453,387.3896$ & \pm 0.0005 & 2644.0 & 0.00065 & I & Hübscher et al. (2005) \\
\hline $2,453,388.6336$ & \pm 0.0001 & 2645.0 & 0.00027 & I & Smith \& Caton (2007) \\
\hline $2,453,407.2977$ & \pm 0.0006 & 2660.0 & -0.00139 & I & Hübscher et al. (2005) \\
\hline $2,453,407.2987$ & \pm 0.0035 & 2660.0 & -0.00039 & $\mathrm{I}$ & Hübscher et al. (2005) \\
\hline $2,453,410.4121$ & \pm 0.0003 & 2662.5 & 0.00205 & II & Hübscher et al. (2005) \\
\hline $2,453,414.1438$ & \pm 0.0009 & 2665.5 & 0.00060 & II & This paper (SOAO) \\
\hline $2,453,425.3416$ & \pm 0.0001 & 2674.5 & -0.00106 & II & Wolf et al. (2007) \\
\hline $2,453,641.2436$ & \pm 0.0003 & 2848.0 & 0.00016 & I & This paper (CbNUO) \\
\hline $2,453,675.4626$ & \pm 0.0003 & 2875.5 & -0.00144 & II & Bíró et al. (2007) \\
\hline $2,453,694.7519$ & \pm 0.0002 & 2891.0 & -0.00012 & $\mathrm{I}$ & Baldwin \& Samolyk (2007) \\
\hline $2,453,704.7068$ & \pm 0.0001 & 2899.0 & -0.00031 & $\mathrm{I}$ & Nelson (2006) \\
\hline $2,453,713.4178$ & \pm 0.0001 & 2906.0 & -0.00001 & I & Wolf et al. (2007) \\
\hline $2,453,746.3945$ & \pm 0.0002 & 2932.5 & 0.00047 & II & Wolf et al. (2007) \\
\hline $2,453,764.4382$ & \pm 0.0005 & 2947.0 & 0.00057 & I & Hübscher et al. (2006) \\
\hline $2,453,769.4149$ & \pm 0.0001 & 2951.0 & -0.00027 & I & Wolf et al. (2007) \\
\hline $2,453,793.0585$ & \pm 0.0001 & 2970.0 & -0.00001 & $\mathrm{I}$ & This paper (CbNUO) \\
\hline $2,454,054.3794$ & \pm 0.0001 & 3180.0 & -0.00029 & $\mathrm{I}$ & Doğru et al. (2007) \\
\hline $2,454,070.5565$ & \pm 0.0002 & 3193.0 & -0.00022 & $\mathrm{I}$ & Wolf et al. (2007) \\
\hline $2,454,084.2455$ & \pm 0.0002 & 3204.0 & 0.00052 & $\mathrm{I}$ & This paper (CbNUO) \\
\hline $2,454,085.4892$ & \pm 0.0006 & 3205.0 & -0.00017 & I & Hübscher \& Walter (2007) \\
\hline $2,454,115.3547$ & \pm 0.0010 & 3229.0 & 0.00004 & $\mathrm{I}$ & Hübscher (2007) \\
\hline $2,454,120.9528$ & \pm 0.0005 & 3233.5 & -0.00160 & II & This paper (CbNUO) \\
\hline $2,454,141.4868$ & \pm 0.0002 & 3250.0 & 0.00002 & I & Wolf et al. (2007) \\
\hline $2,454,152.6860$ & \pm 0.0001 & 3259.0 & -0.00027 & $\mathrm{I}$ & Baldwin \& Samolyk (2007) \\
\hline $2,454,171.3512$ & \pm 0.0009 & 3274.0 & -0.00088 & $\mathrm{I}$ & Hübscher (2007) \\
\hline $2,454,171.3516$ & \pm 0.0001 & 3274.0 & -0.00048 & I & Wolf et al. (2007) \\
\hline $2,454,176.3298$ & \pm 0.0001 & 3278.0 & 0.00018 & $\mathrm{I}$ & Wolf et al. (2007) \\
\hline $2,454,186.2843$ & \pm 0.0002 & 3286.0 & -0.00042 & $\mathrm{I}$ & Wolf et al. (2007) \\
\hline $2,454,487.4260$ & \pm 0.0001 & 3528.0 & -0.00045 & $\mathrm{I}$ & Borkovits et al. (2008) \\
\hline
\end{tabular}


Table 3-Continued

\begin{tabular}{lllllll}
\hline \hline \multicolumn{1}{c}{ HJD } & Error & Epoch & $O-C_{\text {full }}$ & Min & \multicolumn{1}{c}{ References } \\
\hline $2,454,501.11462$ & \pm 0.00007 & 3539.0 & -0.00009 & I & This paper (CbNUO) \\
$2,454,506.09212$ & \pm 0.00007 & 3543.0 & -0.00014 & I & This paper (CbNUO) \\
$2,454,510.4465$ & \pm 0.0003 & 3546.5 & -0.00112 & II & Borkovits et al. (2008) \\
$2,454,558.3573$ & \pm 0.0003 & 3585.0 & 0.00077 & I & Brát et al. (2008) \\
$2,454,815.94500$ & \pm 0.00007 & 3792.0 & 0.00029 & I & This paper (CbNUO) \\
$2,454,834.6136$ & \pm 0.0001 & 3807.0 & 0.00308 & I & Brát et al. (2009) \\
$2,454,843.3212$ & \pm 0.0002 & 3814.0 & -0.00003 & I & Brát et al. (2009) \\
\hline
\end{tabular}

Table 4. Parameters for the quadratic plus LTT ephemeris of CL Aur.

\begin{tabular}{|c|c|c|}
\hline Parameter & Value & Unit \\
\hline$T_{0}$ & $2,450,097,27082 \pm 0.00046$ & HJD \\
\hline$P$ & $1.24437498 \pm 0.00000017$ & $\mathrm{~d}$ \\
\hline$A$ & $(2.44 \pm 0.34) \times 10^{-10}$ & $\mathrm{~d}$ \\
\hline$a_{12} \sin i_{3}$ & $2.38 \pm 0.12$ & $\mathrm{AU}$ \\
\hline$\omega$ & $218.9 \pm 2.7$ & deg \\
\hline$e$ & $0.337 \pm 0.053$ & \\
\hline$n$ & $0.04556 \pm 0.00029$ & $\operatorname{deg} d^{-1}$ \\
\hline$T$ & $2,444,072 \pm 56$ & HJD \\
\hline$P_{3}$ & $21.63 \pm 0.14$ & yr \\
\hline$K$ & $0.01330 \pm 0.00069$ & $\mathrm{~d}$ \\
\hline$f\left(M_{3}\right)$ & $0.0290 \pm 0.0015$ & $M_{\odot}$ \\
\hline$M_{3}\left(i_{3}=90 \mathrm{deg}\right)^{\dagger}$ & 0.83 & $M_{\odot}$ \\
\hline$M_{3}\left(i_{3}=60 \mathrm{deg}\right)^{\dagger}$ & 0.98 & $M_{\odot}$ \\
\hline$M_{3}\left(i_{3}=30 \mathrm{deg}\right)^{\dagger}$ & 1.92 & $M_{\odot}$ \\
\hline$d P / d t$ & $(1.43 \pm 0.20) \times 10^{-7}$ & $\mathrm{~d} \mathrm{yr}^{-1}$ \\
\hline$d M / d t$ & $1.30 \times 10^{-7}$ & $M_{\odot} \mathrm{yr}^{-1}$ \\
\hline
\end{tabular}

${ }^{\dagger}$ Masses of the third body for different values of $i_{3}$. 
Table 5. Applegate-model parameters for the cool secondary of CL Aur.

\begin{tabular}{lcc}
\hline \hline Parameter & Value & Unit \\
\hline$\Delta P$ & 1.1372 & $\mathrm{~s}$ \\
$\Delta P / P$ & $1.06 \times 10^{-5}$ & \\
$\Delta Q$ & $8.49 \times 10^{50}$ & $\mathrm{~g} \mathrm{~cm}^{2}$ \\
$\Delta J$ & $1.46 \times 10^{48}$ & $\mathrm{~g} \mathrm{~cm}^{2} \mathrm{~s}^{-1}$ \\
$I_{\mathrm{s}}$ & $5.47 \times 10^{54}$ & $\mathrm{~g} \mathrm{~cm}^{2}$ \\
$\Delta \Omega$ & $2.68 \times 10^{-7}$ & $\mathrm{~s}^{-1}$ \\
$\Delta \Omega / \Omega$ & $4.59 \times 10^{-3}$ & \\
$\Delta E$ & $7.85 \times 10^{41}$ & $\mathrm{erg}^{-1}$ \\
$\Delta L_{\mathrm{rms}}$ & $3.61 \times 10^{33}$ & $\mathrm{erg} \mathrm{s}^{-1}$ \\
& 0.926 & $L_{\odot}$ \\
$\Delta m_{\mathrm{rms}}$ & 0.103 & $L_{2}$ \\
$B$ & \pm 0.018 & $\mathrm{mag}$ \\
\hline
\end{tabular}


Table 6. Photometric solutions of CL Aur.

\begin{tabular}{|c|c|c|c|c|}
\hline \multirow[t]{2}{*}{ Parameter } & \multicolumn{2}{|c|}{ Without spot } & \multicolumn{2}{|c|}{ With spot } \\
\hline & Primary & Secondary & Primary & Secondary \\
\hline$T_{0}$ (HJD) & \multicolumn{2}{|c|}{$2,452,974.25441 \pm 0.00006$} & \multicolumn{2}{|c|}{$2452974.25438 \pm 0.00005$} \\
\hline$P(\mathrm{~d})$ & \multicolumn{2}{|c|}{$1.24438169 \pm 0.00000025$} & \multicolumn{2}{|c|}{$1.24438175 \pm 0.00000025$} \\
\hline$q$ & \multicolumn{2}{|c|}{$0.5944(55)$} & \multicolumn{2}{|c|}{$0.6023(49)$} \\
\hline$i(\mathrm{deg})$ & \multicolumn{2}{|c|}{$88.15(11)$} & \multicolumn{2}{|c|}{$88.21(12)$} \\
\hline$T(\mathrm{~K})$ & 9,420 & $6,315(46)$ & 9,420 & $6,323(44)$ \\
\hline$\Omega$ & $3.569(14)$ & 3.053 & $3.563(13)$ & 3.068 \\
\hline$A$ & 1.0 & 0.5 & 1.0 & 0.5 \\
\hline$g$ & 1.0 & 0.32 & 1.0 & 0.32 \\
\hline$X$ & 0.612 & 0.478 & 0.612 & 0.478 \\
\hline$x_{B}$ & $0.601(28)$ & $0.375(103)$ & $0.585(27)$ & $0.368(101)$ \\
\hline$x_{V}$ & $0.515(30)$ & $0.539(70)$ & $0.511(27)$ & $0.538(68)$ \\
\hline$x_{R}$ & $0.408(31)$ & $0.528(54)$ & $0.409(28)$ & $0.529(53)$ \\
\hline$x_{I}$ & $0.329(30)$ & $0.397(45)$ & $0.332(28)$ & $0.398(44)$ \\
\hline$L /\left(L_{1}+L_{2}\right)_{B}$ & $0.8629(24)$ & 0.1371 & $0.8630(22)$ & 0.1370 \\
\hline$L /\left(L_{1}+L_{2}\right)_{V}$ & $0.8163(24)$ & 0.1837 & $0.8163(22)$ & 0.1837 \\
\hline$L /\left(L_{1}+L_{2}\right)_{R}$ & $0.7683(25)$ & 0.2317 & $0.7683(23)$ & 0.2317 \\
\hline$L /\left(L_{1}+L_{2}\right)_{I}$ & $0.7185(26)$ & 0.2815 & $0.7185(23)$ & 0.2815 \\
\hline$r$ (pole) & $0.3327(17)$ & $0.3135(7)$ & $0.3343(15)$ & $0.3145(7)$ \\
\hline$r$ (point) & $0.3643(26)$ & $0.4467(29)$ & $0.3671(24)$ & $0.4481(25)$ \\
\hline$r($ side $)$ & $0.3432(19)$ & $0.3276(8)$ & $0.3451(17)$ & $0.3287(7)$ \\
\hline$r$ (back) & $0.3547(20)$ & $0.3598(8)$ & $0.3569(20)$ & $0.3609(7)$ \\
\hline 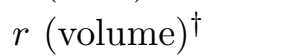 & 0.3439 & 0.3350 & 0.3458 & 0.3361 \\
\hline Colatitude (deg) & & & $71.87(85)$ & \\
\hline Longitude (deg) & & & $2.36(29)$ & \\
\hline Radius (deg) & & & 14.14(18) & \\
\hline$T_{\text {spot }} / T_{\text {local }}$ & & & $1.125(9)$ & \\
\hline$\Sigma W(O-C)^{2}$ & & 12 & & \\
\hline
\end{tabular}


Table 7. Minimum timings of CL Aur determined by two different methods.

\begin{tabular}{cccccc}
\hline \hline KvW & W-D & Error $^{\mathrm{a}}$ & Difference $^{\mathrm{b}}$ & Filter & Min \\
\hline $2,452,974.2543$ & $2,452,974.25469$ & \pm 0.00007 & -0.00039 & $B V R I$ & $\mathrm{I}$ \\
$2,453,019.0518$ & $2,453,019.05186$ & \pm 0.00010 & -0.00006 & $B V R I$ & $\mathrm{I}$ \\
$2,453,055.1393$ & $2,453,055.13934$ & \pm 0.00010 & -0.00004 & $B V R I$ & $\mathrm{I}$ \\
$2,453,350.0577$ & $2,453,350.05766$ & \pm 0.00005 & +0.00004 & $B V R I$ & $\mathrm{I}$ \\
$2,453,353.1692$ & $2,453,353.16967$ & \pm 0.00031 & -0.00047 & $B V R I$ & $\mathrm{II}$ \\
$2,453,360.0128$ & $2,453,360.01267$ & \pm 0.00009 & +0.00013 & $B V R I$ & $\mathrm{I}$ \\
$2,453,361.2570$ & $2,453,361.25700$ & \pm 0.00009 & +0.00000 & $B V R I$ & $\mathrm{I}$ \\
$2,453,414.1438$ & $2,453,414.14313$ & \pm 0.00042 & +0.00067 & $B V R I$ & $\mathrm{II}$ \\
\hline
\end{tabular}

${ }^{a}$ Errors yielded by the W-D code.

${ }^{\mathrm{b}}$ Differences between columns (1) and (2).

Table 8. Astrophysical parameters for CL Aur.

\begin{tabular}{lcc}
\hline \hline \multicolumn{1}{c}{ Parameter } & Primary & Secondary \\
\hline$M / M_{\odot}$ & 2.24 & 1.35 \\
$R / R_{\odot}$ & 2.58 & 2.51 \\
$\log g(\mathrm{cgs})$ & 3.97 & 3.77 \\
$\log \rho / \rho_{\odot}$ & -0.88 & -1.07 \\
$T(\mathrm{~K})$ & 9,420 & 6,323 \\
$L / L_{\odot}$ & 46.96 & 9.02 \\
$B-V(\mathrm{mag})$ & -0.01 & +0.48 \\
$M_{\mathrm{bol}}(\mathrm{mag})$ & +0.51 & +2.30 \\
$\mathrm{BC}(\mathrm{mag})$ & -0.15 & -0.01 \\
$M_{V}(\mathrm{mag})$ & +0.66 & +2.31 \\
$M_{\mathrm{V}, \text { total }}^{\dagger}(\mathrm{mag})$ & \multicolumn{2}{c}{+0.44} \\
Distance $(\mathrm{pc})$ & \multicolumn{2}{c}{1,150} \\
\hline \multicolumn{3}{c}{}
\end{tabular}

${ }^{\dagger}$ Absolute visual magnitude from both components. 
Table 9. Impact position and energy of transferred matter.

\begin{tabular}{lcccccc}
\hline \hline \multicolumn{2}{c}{ Initial velocity $\left(\mathrm{km} \mathrm{s}^{-1}\right)$} & & \multicolumn{2}{c}{ Impact position $(\mathrm{deg})$} & Impact energy $\left(L_{\odot}\right)$ \\
\cline { 1 - 2 } \cline { 5 - 6 }$v_{x}$ & $v_{y}$ & $v_{z}$ & & Longitude & Colatitude & \\
\cline { 1 - 2 } $3 \times 10^{-7}$ & 0 & 0 & & 10.5 & 90.0 & 0.69 \\
$3 \times 10^{-5}$ & 0 & 0 & & 10.3 & 90.0 & 0.69 \\
$3 \times 10^{-3}$ & 0 & 0 & & 10.5 & 90.0 & 0.69 \\
$3 \times 10^{-1}$ & 0 & 0 & & 10.6 & 90.0 & 0.70 \\
3 & 0 & 0 & & 10.5 & 90.0 & 0.71 \\
30 & 0 & 0 & & 11.3 & 90.0 & 0.73 \\
300 & 0 & 0 & & 4.9 & 90.0 & 1.85 \\
450 & 0 & 0 & & 3.4 & 90.0 & 3.24 \\
$3 \times 10^{-1}$ & 0 & $3 \times 10^{-1}$ & 10.6 & 90.0 & 0.70 \\
3 & 0 & 3 & & 10.6 & 90.3 & 0.71 \\
30 & 0 & 30 & & 11.4 & 85.3 & 0.72 \\
150 & 0 & 150 & 12.4 & 65.4 & 1.26 \\
300 & 0 & 300 & 20.6 & 43.6 & 2.96 \\
\hline
\end{tabular}

